\title{
Plantations d'eucalyptus et sidérurgie en Amazonie : apports du modèle 3-PG
}

Maurel BEHLING ${ }^{1}$

Marie Gabrielle PIKETTY2

Thiago Fonseca MORELLO3

Jean-Pierre BouILLET $T^{4,5}$

Franscisco MESQUITA NeTO ${ }^{6}$

Jean-Paul LACLAU 4,5

${ }^{1}$ Embrapa Agrossilvipastoril

Caixa Postal 343

Sinop-MT

Cep 78.550-970

Brésil

${ }^{2}$ Cirad

Upr Green

TA-C47/F

Campus international de Baillarguet 34398 Montpellier Cedex 5

France

${ }^{3}$ Faculdade de Economia Administração e Contabilidade da Universidade Usp-Nesa

São Paulo

Brésil

${ }^{4}$ ESALQ, USP, Departamento de Ciências Florestais

Avenida Pàdua Dias, 11

Caixa Postal 9-13418-900

Piracicaba

Brésil

5 Cirad

Umr Eco\&Sols

Campus de la Gaillarde

Bât. 12 - 2, place Viala

34060 Montpellier Cedex 2

France

${ }^{6}$ Dow Corning Metais do Pará Indústria e Comércio Ltda

Rodovia PA 263, km 3,5 s/n

Breu Branco, PA, Cep : 68488-000

Brésil

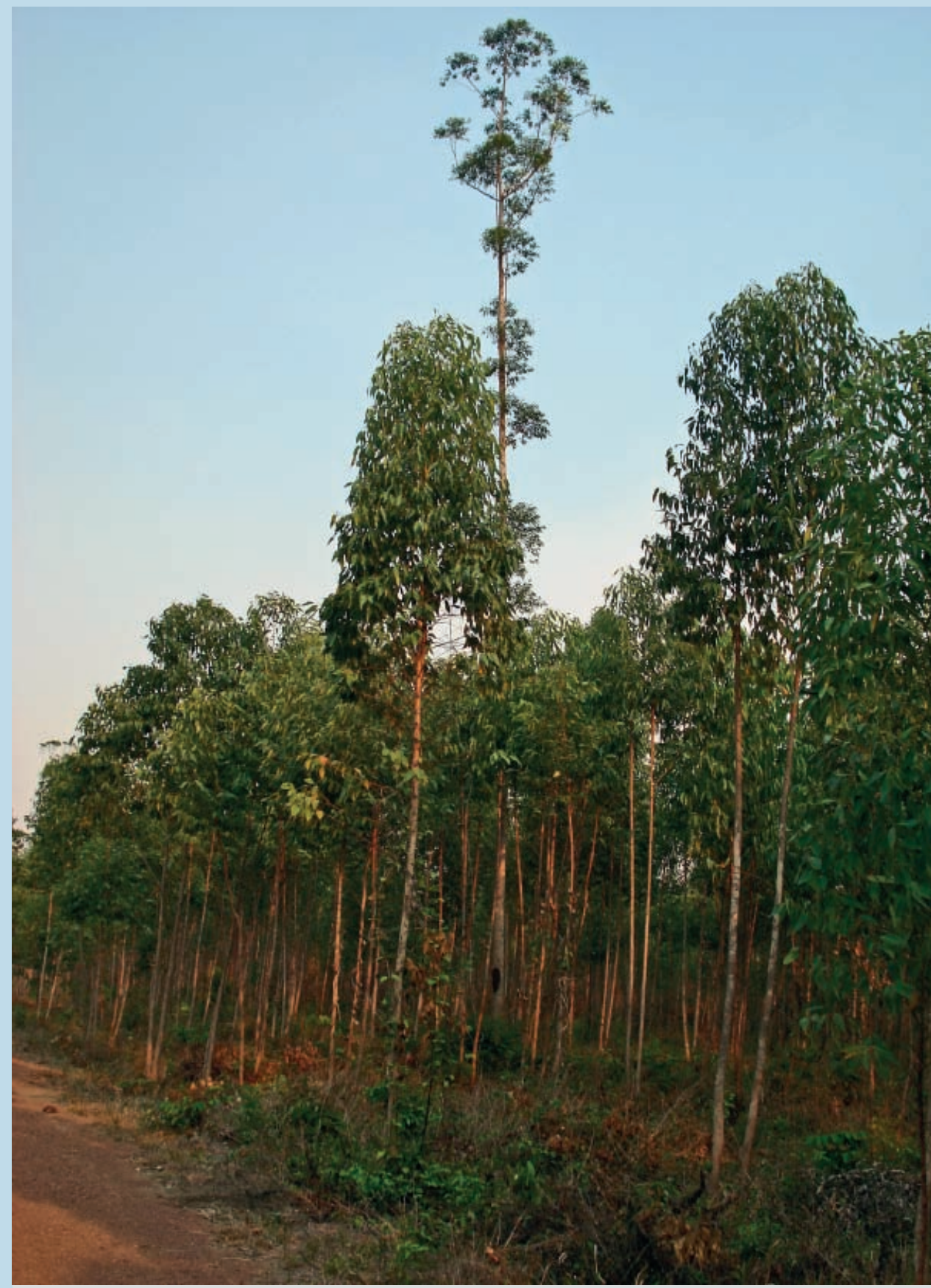

Taillis d'eucalyptus âgé d'environ un an. Un arbre de la rotation précédente apparait en arrière-plan.

Photo M. Behling. 


\section{RÉSUMÉ}

\section{PLANTATIONS D'EUCALYPTUS ET SIDÉRURGIE EN AMAZONIE : APPORTS DU MODÈLE 3-PG}

Le secteur sidérurgique de Carajás, en Amazonie Brésilienne, fait l'objet de contestations environnementales du fait de sa forte consommation en charbon de bois. Ce charbon est essentiellement produit à partir de biomasse de forêts naturelles, avec des impacts directs et indirects sur la déforestation et la dégradation de ces écosystèmes. Les plantations d'eucalyptus à vocation énergétique installées sur des pâturages dégradés constituent une alternative intéressante. Peu de ces plantations ont été mises en place jusqu'à présent et faute d'évaluations valables de leur potentiel de production, une étude a été menée pour présenter une estimation consolidée de la croissance et de la productivité des plantations d'eucalyptus du pôle de Carajás. Celles-ci ont été obtenues à partir du modèle 3-PG (Physiological Principles in Predicting Growth). Son paramétrage a été réalisé à partir de données de croissance du massif d'eucalyptus d'une entreprise de la municipalité de Breu Branco dans l'État du Pará au Brésil. La calibration locale du modèle s'est avérée beaucoup plus performante que l'utilisation de paramètres établis pour des plantations d'eucalyptus dans d'autres régions du Brésil, d'Afrique du sud ou d'Australie. Les simulations réalisées indiquent que l'accroissement moyen annuel actuel de l'ordre de $20 \mathrm{~m}^{3}$ à l'hectare, obtenu pour une période de six ans, pourrait augmenter jusqu'à $30 \mathrm{~m}^{3}$ avec des fertilisations adaptées et un contrôle efficace du sous bois. Elles suggèrent aussi que des productions supérieures seraient obtenues sans déficit hydrique. Cela montre la nécessité de sélectionner les zones de plantation où la saison sèche est la moins marquée autour du pôle de Carajás. La calibration du modèle 3-PG a permis d'améliorer cet outil de gestion des plantations industrielles dans les conditions amazoniennes.

Mots-clés : eucalyptus, charbon de bois, bois énergie, modélisation, croissance, productivité, potentiel, sylviculture, Amazonie.

\section{ABSTRACT}

\section{EUCALYPTUS PLANTATIONS AND THE STEEL INDUSTRY IN AMAZONIA - A CONTRIBUTION FROM THE 3-PG MODEL}

The Carajás steel industry sector in the Brazilian Amazon has aroused protest on environmental grounds because of its heavy reliance on charcoal. The charcoal is mainly produced from natural forest biomass, with direct and indirect impacts on deforestation and forest ecosystem degradation. Establishing eucalyptus plantations for fuel on degraded pastures could be a workable alternative. Few such plantations exist as yet, and because there are no validated assessments of their production potential, a study was conducted to provide consolidated estimations of the growth and productivity of the Carajás eucalyptus plantations. The estimations were obtained with the 3-PG model (Physiological Principles in Predicting Growth). The model parameters are based on growth data for the eucalyptus plantations established by a company in Breu Branco municipality in Brazil's Pará State. Calibrating the model with local data proved to be far more effective than using the parameters set for eucalyptus plantations in other areas in Brazil, South Africa or Australia. The simulations made show that the current annual average growth rate, over a six-year period, of about $20 \mathrm{~m}^{3}$ per hectare could increase to $30 \mathrm{~m}^{3}$ with appropriate fertilisation and effective underbrush control. They also suggest that production could be higher without water deficit. Plantation zones shall be selected as a priority in areas where the dry season is the least severe around Carajás. These 3-PG model settings have made it a more effective management tool for industrial plantations in Amazonian conditions.

Keywords: eucalyptus, charcoal, fuelwood, modelling, growth, productivity, potential, silviculture, Amazonia

\section{RESUMEN}

\section{PLANTACIONES DE EUCALIPTO Y SIDERURGIA EN LA AMAZONÍA: APORTES DEL MODELO 3-PG}

El sector siderúrgico de Carajás, en la Amazonia brasileña, es objeto de protestas ambientales por su alto consumo de carbón vegetal. Este carbón se produce fundamentalmente a partir de biomasa de bosques naturales, con impactos directos e indirectos en la deforestación y degradación de estos ecosistemas. Las plantaciones de eucaliptos para fines energéticos establecidas en pastos degradados suponen una alternativa interesante. Hasta ahora sólo se han establecido unas cuantas plantaciones de este tipo $y$, al no existir evaluaciones válidas de su potencial de producción, se ha llevado a cabo un estudio para presentar una estimación consolidada del crecimiento y productividad de las plantaciones de eucaliptos del polo siderúrgico de Carajás. Dichos valores se obtuvieron a partir del modelo 3-PG (Physiological Principles in Predicting Growth). La parametrización se efectuó con los datos de crecimiento del rodal de eucaliptos de una empresa del municipio de Breu Branco, en el Estado brasileño de Pará. La calibración local del modelo resultó ser mucho más eficiente que el uso de parámetros establecidos para plantaciones de eucaliptos de otras regiones de Brasil, Sudáfrica o Australia. Las simulaciones realizadas indican que el incremento promedio anual actual del orden de $20 \mathrm{~m}^{3}$ por hectárea, obtenido para un período de seis años, podría aumentar hasta $30 \mathrm{~m}^{3}$ con fertilizaciones adaptadas y un control eficaz del sotobosque. Dichas simulaciones también sugieren que se podrían lograr producciones superiores sin déficit hídrico. Esto muestra la necesidad de seleccionar las zonas idóneas para plantaciones donde la época seca es la menos intensa, alrededor del polo de Carajás. La calibración del modelo 3-PG permitió mejorar esta herramienta de manejo de plantaciones industriales en condiciones amazónicas.

Palabras clave: eucalipto, carbón vegetal, dendroenergía, modelización, crecimiento, productividad, potencial, silvicultura, Amazonia. 


\section{Introduction}

Disposant d'importants gisements de minerai de fer, dans la région du Minas Gerais et sur le pôle de Carajás en Amazonie, le Brésil est le neuvième producteur mondial d'acier brut avec une production de 26,5 millions de tonnes en 2009. Une des spécificités du secteur sidérurgique brésilien est qu'il utilise de manière importante le charbon de bois comme agent thermoréducteur. Ainsi, environ $35 \%$ de la production du secteur sidérurgique brésilien utilisait le charbon de bois en 2006 (SINDIfER, 2010), le Brésil étant aujourd'hui le seul pays où le secteur sidérurgique utilise encore du charbon de bois à grande échelle.

Sur le pôle de Carajás, en Amazonie brésilienne (figure 1), où est produit un peu plus d'un tiers de la production nationale de fonte indépendante, les entreprises utilisent exclusivement du charbon de bois. La demande annuelle est d'environ 2,3 millions de tonnes de charbon dont $73 \%$ serait fabriqué à partir de biomasse de forêts naturelles, le complément provenant de plantations d'eucalyptus (CALAIS, 2009). Selon PIKETTY et al. (2011), ce serait un peu moins de 20 \% du charbon de bois du pôle qui proviendrait de plantations.

L'utilisation de charbon de bois pour produire de la fonte présente des atouts environnementaux par rapport au charbon minéral, lorsque la biomasse utilisée n'est pas issue de la déforestation ou de la dégradation de forêts naturelles. La plus grande partie du bois employé sur le pôle de Carajás pour la production de charbon provient de résidus principalement récupérés dans des scieries après transformation des grumes ou, dans une proportion beaucoup plus faible, d'exploitation forestière (les résidus sont alors collectés directement au sein des surfaces exploitées). Une partie du bois provient également de la déforestation de parcelles à vocation agricole. Il est difficile de réaliser un diagnostic précis de l'origine du bois utilisé pour produire du charbon. Les seules informations certaines sont la part majeure provenant de bois issus de forêts exploitées illégalement, une faible proportion provenant de plantations forestières, et une part mineure de résidus d'exploitation de forêts naturelles commercialisés avec autorisation (PIKETTY et al., 2011). Le bois issu de forêts naturelles représenterait donc aujourd'hui une source de biomasse importante pour la fabrication du charbon utilisé sur le pôle de Carajas, ce qui contribue à accroître les pressions existant sur les forêts naturelles.

Le secteur sidérurgique du pôle fait l'objet de contestations environnementales fortes et il se trouve confronté à un enjeu de taille : prouver que la biomasse utilisée pour produire le charbon de bois qu'il consomme ne participe pas à la déforestation de l'Amazonie. Dans ce cadre, la reforestation des terres dégradées, qui couvrent des surfaces importantes autour du pôle, est une des alternatives potentiellement intéressantes pour le secteur sidérurgique afin de faire face aux contestations environnementales.

Un premier diagnostic réalisé dans la région montre que l'essentiel des plantations forestières énergétiques est constitué de plantations d'eucalyptus (BoulLLET et al., 2010). Il existe également de nombreuses initiatives de

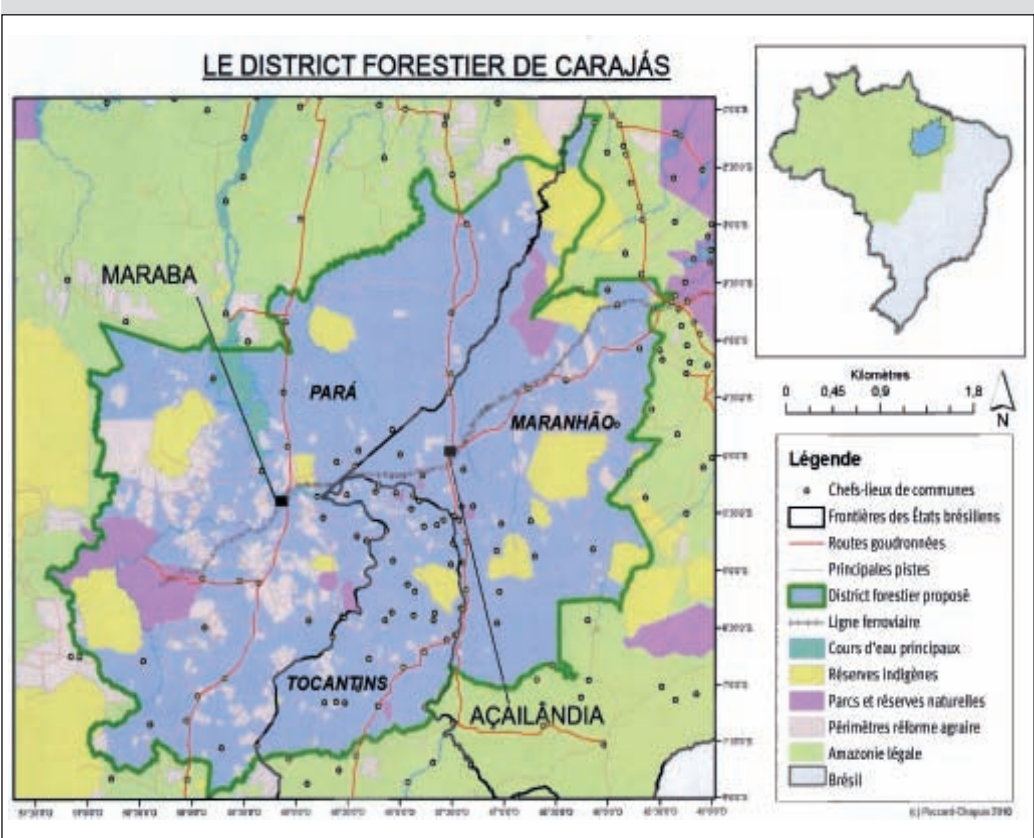

Figure 1.

Le district forestier de Carajás.

plantations d'espèces issues de la forêt naturelle amazonienne. II est aujourd'hui impossible de faire une analyse détaillée de leurs potentialités pour la production de charbon de bois : très peu de peuplements avec ces espèces ont atteint un âge proche de l'exploitation et les suivis de croissance sont trop rares pour évaluer correctement les itinéraires techniques et leurs impacts sur la productivité (PIKetTy et al., 2011). En outre, dans l'état actuel des connaissances et après plus d'un siècle d'introduction d'espèces forestières exotiques en zone tropicale, ce sont les eucalyptus qui remplissent le mieux l'ensemble des critères d'aptitude technique à la production de charbon de bois : rusticité, production élevée et caractéristiques technologiques adaptées (BoulLLET et al., 2010).

Une analyse a été menée pour évaluer le potentiel des plantations d'eucalyptus dans la région du pôle sidérurgique de Carajás ${ }^{1}$. En effet, la gestion durable des plantations forestières nécessite une connaissance précise de la ressource et des prévisions fiables de production sur la durée de la rotation. Ces informations sont indispensables pour dimensionner les infrastructures (routes, tracteurs, main-d'œuvre, usines...) par rapport au potentiel de production et modifier la sylviculture quand la croissance des arbres s'avère inférieure aux prévisions. Cette étude présente des estimations de croissance et de productivité de plantations d'eucalyptus sur le pôle de Carajás, obtenues à partir de la calibration du modèle 3-PG (Physiological Principles in Predicting Growth) pour les plantations existant dans la région. Après avoir décrit la méthodologie retenue, les résultats du modèle calibré sont présentés ainsi que les enseignements de cette approche de modélisation pour la sélection des zones à planter et la gestion des massifs forestiers industriels. 


\section{Méthodologie}

\section{Choix du modèle}

Des modèles mécanistes (fondés sur des processus écophysiologiques) ont été conçus pour estimer la productivité de peuplements forestiers au cours des vingt dernières années. De tels modèles constituent un outil d'analyse des effets de scénarios contrastés de conditions de croissance en fonction du climat, du sol, du type de matériel végétal et de la sylviculture appliquée. Ils peuvent permettre ainsi d'optimiser plusieurs facteurs de production afin d'améliorer la croissance des arbres et de prévoir les impacts de la sylviculture sur l'environnement. Cependant, le grand nombre de paramètres à renseigner, du fait de la complexité des processus physiologiques à prendre en compte, est un obstacle important à l'utilisation pratique de ces modèles par les gestionnaires forestiers (BATTAGLIA, SANDS, 1997).

Le modèle 3-PG est basé sur l'efficience d'utilisation des radiations solaires et de l'eau pour l'allocation du carbone dans les compartiments des arbres. Cet outil s'avère intéressant pour des plantations forestières à rotations courtes afin de tenir compte de l'impact de la variabilité du climat sur la production. Il a été développé pour estimer la productivité de peuplements forestiers monospécifiques équiennes et peut être considéré comme un modèle hybride qui incorpore à la fois des relations empiriques obtenues à partir de mesures expérimentales et des processus physiologiques utilisés dans des modèles de bilan carboné. La performance du modèle 3-PG pour estimer la productivité de forêts commerciales a été montrée en Australie, Nouvelle-Zélande, aux États-Unis et en Afrique du Sud (e.g.

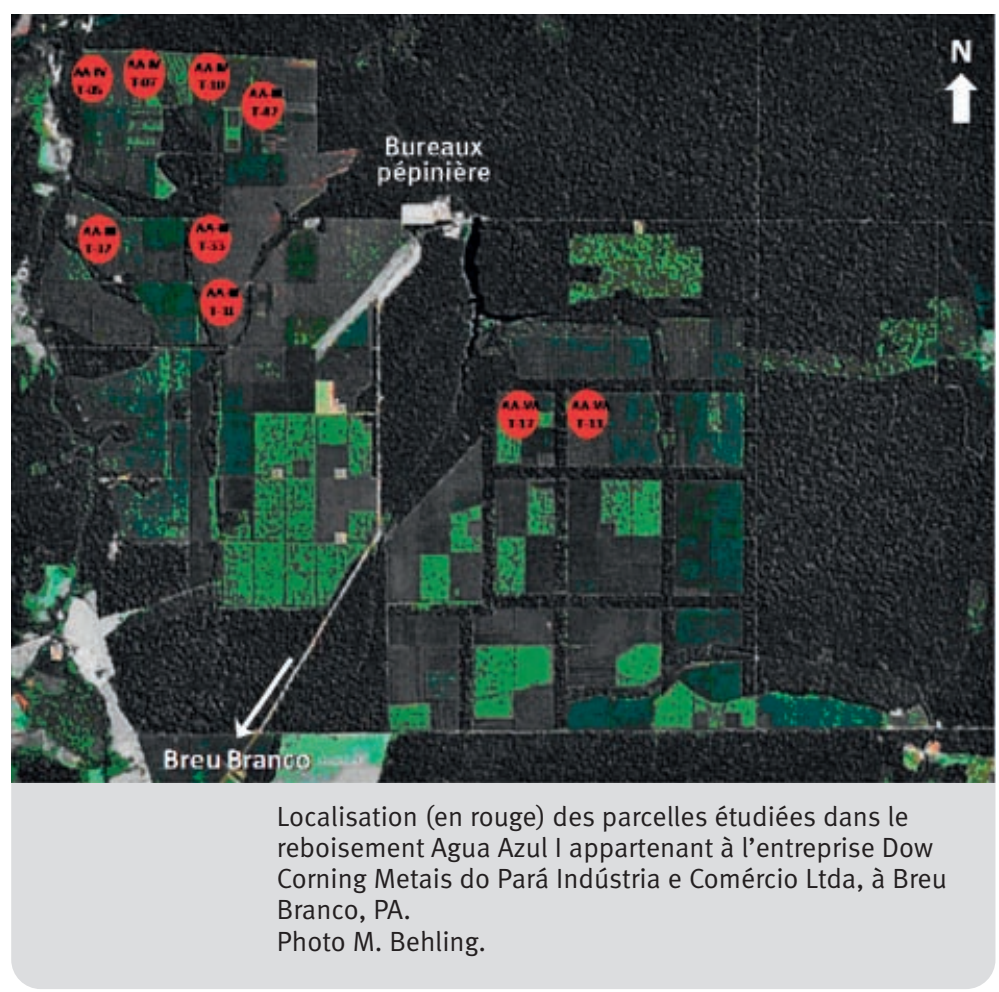

COOPS, WARING, 2001 ; DYE, 2004). Ce modèle a aussi été utilisé pour des plantations commerciales d'eucalyptus situées dans plusieurs États du Brésil (STAPE et al., 2004 ; ALMEIDA et al., 2004).

De manière générale, ces travaux mettent en évidence le potentiel de 3-PG comme outil d'aide à l'aménagement forestier. Ce modèle permet de prévoir l'impact des facteurs déterminants de la productivité (radiation solaire, température, eau et nutriments) sur le potentiel de production. Les estimations fournies par le modèle 3-PG sont sensibles à différents facteurs qui doivent être calibrés avec précision pour la zone d'étude : la relation entre les productions primaires nette et brute, l'efficience du feuillage à assimiler le carbone à partir des radiations incidentes, l'aire foliaire spécifique, la conductance du houppier, le taux de chute de litière, les coefficients des équations allométriques adaptés au matériel végétal étudié et enfin la densité du bois (ESPREY et al., 2004).

Ce modèle permet une prise en compte simplifiée de processus physiologiques complexes tout en diminuant le nombre de variables d'entrée (LANDSBERG et al., 2003). Son équation générale est la suivante :

$G P P=A P A R \times L \times F_{\text {eau }} \times F_{\text {nutriments }} \times F_{V P D}$

où GPP est la production primaire brute, APAR est le rayonnement photosynthétiquement actif absorbé, L est la photosynthèse potentielle maximale, $F_{\text {eau }}$ est un réducteur de la production primaire lié à la disponibilité hydrique, $F_{\text {nutriments }}$ est un réducteur de la production primaire lié à la disponibilité en nutriments et $F_{V P D}$ est un réducteur de la production primaire.

Les paramètres de ce modèle ont été déterminés dans les conditions de sol et de climat de la région du pôle sidérurgique de Carajás, afin d'évaluer le potentiel de production du matériel végétal utilisé et d’identifier les principaux facteurs qui contrôlent la croissance des eucalyptus dans cette zone. Les procédures utilisées pour la paramétrisation et la calibration du modèle ont été similaires à celles décrites par LANDSBERG et al. (2003). La version du modèle utilisée a été la version 3PGpjs2.4, mise en œuvre en macros du Visual Basic, en feuilles de calcul Microsoft Excel (SANDS, 2004). La plupart des paramètres des équations utilisées dans le modèle ont été mesurés localement. Les paramètres non mesurés ont été obtenus à partir de travaux réalisés au Brésil avec 3-PG sur l'eucalyptus (ALMEIDA et al., 2004 ; STAPE et al., 2004).

\section{Paramétrisation du modèle 3-PG}

L'étude a été conduite sur un site appartenant à l'entreprise Dow Corning Metais do Pará Indústria e Comércio Ltda, dans les parcelles de reboisement Água Azul I (tableau I). Ces plantations se trouvent dans la commune de Breu Branco, située dans la région Sud-Ouest de l'État du Pará. Elles ont été installées sur des pâturages dégradés. L'altitude est d'environ $85 \mathrm{~m}$, les précipitations moyennes annuelles de 2360 millimètres $(\mathrm{mm}$ ) et l'humidité moyenne de l'air de $86 \%$. La température moyenne mensuelle varie peu durant l'année (entre $24,3^{\circ} \mathrm{C}$ et $26,9^{\circ} \mathrm{C}$ ). 
Tableau I.

Propriétés de la couche superficielle de sol $(0-40 \mathrm{~cm})$ dans les parcelles échantillonnées pour la calibration du modèle 3-PG.

\begin{tabular}{|c|c|c|c|c|c|c|c|c|c|c|c|c|c|c|c|c|c|}
\hline \multirow[t]{2}{*}{ Clone } & \multirow{2}{*}{$\begin{array}{l}\text { Âge } \\
\text { mois }\end{array}$} & \multirow[t]{2}{*}{ Topographie } & \multicolumn{3}{|c|}{ Granulométrie } & \multirow{2}{*}{$\begin{array}{c}\mathrm{pH} \\
\mathrm{CaCl}_{2}\end{array}$} & \multirow{2}{*}{$\begin{array}{l}\mathrm{MO} \\
\mathrm{g} / \mathrm{kg}\end{array}$} & $\mathrm{P}^{1}$ & S & \multirow[t]{2}{*}{ K } & \multirow[t]{2}{*}{$\mathrm{Ca}$} & $\mathrm{Mg}$ & \multicolumn{2}{|c|}{$\mathrm{Al} \mathrm{H}+\mathrm{Al}$} & \multirow[t]{2}{*}{$\mathrm{SB}^{2}$} & \multirow[t]{2}{*}{ ECEC } & $\mathrm{V}^{3}$ \\
\hline & & & Sable & Limon & Argile & & & & $\mathrm{kg}$ & & & & $\mathrm{mol}_{\mathrm{c}} / \mathrm{k}$ & & & & $\%$ \\
\hline \multirow[t]{5}{*}{ UR-57 } & 12 & Plane & 549 & 13 & 439 & 3,70 & 7,5 & 1,5 & 10,0 & 0,2 & 4,5 & 1,5 & 10,0 & 38,0 & 6,2 & 44,2 & 14,0 \\
\hline & 23 & Plane & 509 & 14 & 477 & 3,80 & 9,0 & 2,5 & 8,5 & 0,3 & 5,0 & 3,0 & 8,0 & 40,0 & 8,3 & 48,3 & 17,0 \\
\hline & 36 & Plane & 568 & 19 & 414 & 3,85 & 6,5 & 2,0 & 7,5 & 0,3 & 5,5 & 1,5 & 8,5 & 36,0 & 7,3 & 43,3 & 16,5 \\
\hline & 46 & Semi-plane & 586 & 13 & 401 & 3,75 & 7,5 & 1,5 & 7,0 & 0,2 & 4,5 & 1,5 & 10,0 & 42,0 & 6,2 & 48,2 & 12,5 \\
\hline & 67 & Semi-plane & 781 & 20 & 200 & 3,75 & 7,5 & 1,5 & 6,5 & 0,1 & 1,0 & 1,0 & 9,5 & 38,0 & 2,1 & 40,1 & 5,5 \\
\hline \multirow[t]{4}{*}{ PE-168 } & 10 & Semi-plane & 747 & 16 & 238 & 3,85 & 7,5 & 1,5 & 9,5 & 0,5 & 5,0 & 1,5 & 7,0 & 32,5 & 7,0 & 39,5 & 17,0 \\
\hline & 20 & Plane & 879 & 21 & 100 & 3,90 & 9,0 & 1,5 & 5,0 & 0,1 & 2,0 & 1,0 & 9,5 & 47,0 & 3,1 & 50,1 & 6,0 \\
\hline & 59 & Plane & 557 & 18 & 426 & 3,90 & 6,5 & 1,0 & 6,5 & 0,2 & 8,5 & 2,0 & 6,0 & 32,5 & 10,7 & 43,2 & 24,5 \\
\hline & 71 & Semi-plane & 837 & 14 & 150 & 4,00 & 8,0 & 1,0 & 7,0 & 0,2 & 1,5 & 1,0 & 8,5 & 31,0 & 2,7 & 33,7 & 8,0 \\
\hline
\end{tabular}

${ }^{1} \mathrm{P}$ extrait par des résines ; ${ }^{2} \mathrm{~K}+\mathrm{Na}+\mathrm{Ca}+\mathrm{Mg} ;{ }^{3}$ Taux de saturation de la CEC effective (ECEC).

Cette étude a été réalisée à partir de deux clones sélectionnés par l'entreprise chez deux espèces d'eucalyptus adaptées aux conditions équatoriales de l'État du Pará : Eucalyptus pellita (clone PE-168) et E. urophylla (clone UR057). Des mesures ont été effectuées dans des parcelles de ces deux clones représentant l'ensemble de la rotation (âgées de 1 à 6 ans), pour deux niveaux de productivité contrastés : une production faible pour le clone PE-168 sur sol sableux et une forte production par rapport à la moyenne du massif pour le clone UR-057 sur sol argileux (tableau I).

Quatre sous-parcelles ont été délimitées dans chacune des neuf parcelles étudiées. Chacune était composée de 100 arbres sur une surface de 870 mètres carrés $\left(\mathrm{m}^{2}\right)$. Le diamètre à 1,30 mètre $(m)$ de hauteur $(D)$ et la hauteur totale de tous les arbres ont été mesurés. À partir de ces inventaires, 10 arbres ont été sélectionnés dans chaque parcelle pour représenter l'amplitude de variation des surfaces terrières (10 classes) puis abattus pour quantifier la biomasse de leurs différents compartiments. Les feuilles, branches, écorce et bois de ces arbres ont été séparés et pesés sur le terrain. Afin de déterminer le poids de matière sèche de chaque compartiment, un échantillon a été pesé à l'état frais pour chaque arbre, puis placé à l'étuve à $65^{\circ} \mathrm{C}$ jusqu'à stabilisation du poids. À partir du poids de matière fraîche totale de chaque compartiment et du pourcentage d'humidité de chaque échantillon, il a été possible d'estimer le poids de matière sèche totale pour chaque compartiment. Des équations allométriques ont ensuite été ajustées pour décrire les relations entre la biomasse ligneuse, le volume du tronc $(\mathrm{V})$, la hauteur totale $(\mathrm{H})$ et le diamètre du tronc à $1,30 \mathrm{~m}$ de hauteur (D).

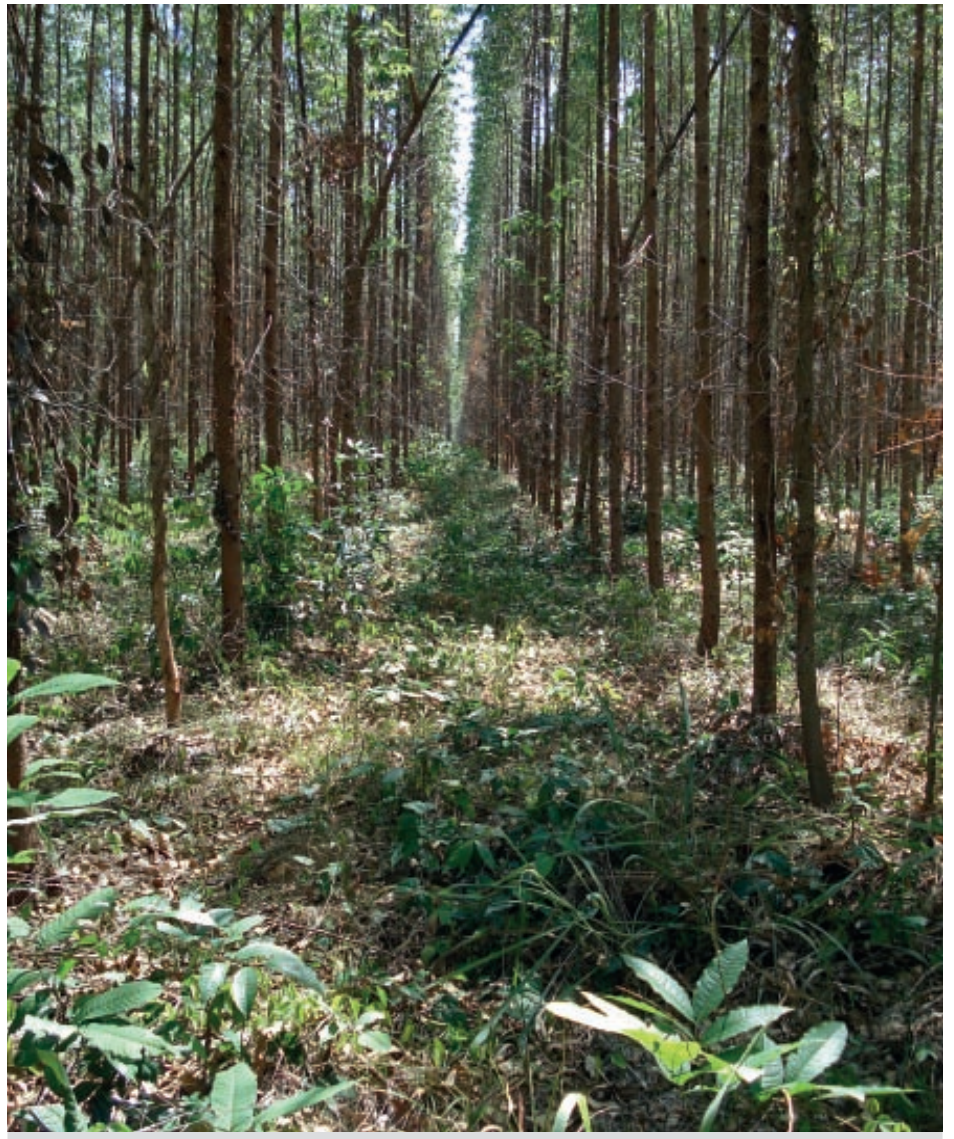

Plantation d'eucalyptus âgée de deux ans environ avec une sylviculture bien adaptée permettant le contrôle de la concurrence herbacée.

Photo M. Behling. 
Tableau II.

Équations ajustées entre l'aire foliaire spécifique (AFS), la densité du bois (d), la fraction de branches et d'écorce parmi la biomasse ligneuse aérienne $\left(f_{B B}\right)$ et l'âge du peuplement pour les deux clones d'eucalyptus étudiés dans le massif de Breu Branco.

\begin{tabular}{|c|c|c|c|}
\hline \multirow[t]{2}{*}{ Clone } & Équation & $\mathrm{n}$ & $\mathrm{R}^{2}$ \\
\hline & Aire foliaire spécifique (AFS) & & \\
\hline UR-057 & AFS $=6,96+(12,086,96) e^{(\ln 2)(a ̂ g e / 1,20)^{2}}$ & 50 & 0,70 \\
\hline PE-168 & AFS $=8,13+(12,838,13) e^{(\ln 2)(a ̂ g e / 1,14)^{2}}$ & 40 & 0,86 \\
\hline UR-057 & $\begin{array}{l}\text { Densité de bois }(d) \\
d=0,57+(0,390,57) e^{(\ln 2)(a ̂ g e / 1,69)}\end{array}$ & 50 & 0,69 \\
\hline \multirow[t]{2}{*}{ PE-168 } & $d=0,54+(0,430,54) e^{(\ln 2)(a ̂ g e / 1,68)}$ & 40 & 0,74 \\
\hline & Fraction de branches et écorce dans la b & se liq̧ & aérienne \\
\hline UR-057 & $f_{b b}=0,16=(0,580,16) e^{(\ln 2)(a ̂ g e / 1,55)^{1,33}}$ & 50 & 0,95 \\
\hline PE-168 & $f_{b b}=0,17+(0,550,16) e^{(\ln 2)(a ̂ g e / 1,70)^{3,24}}$ & 40 & 0,98 \\
\hline
\end{tabular}

AFS ( $\left.\mathrm{m}^{2} / \mathrm{kg}\right)$; âge (années) ; D (tonne par $\left.\mathrm{m}^{3}\right) ; \mathrm{f}_{\mathrm{BB}}(\mathrm{kg} / \mathrm{kg}) ; \mathrm{n}$ : nombre d'observations ; $\mathrm{R}^{2}$ : coefficient de détermination.

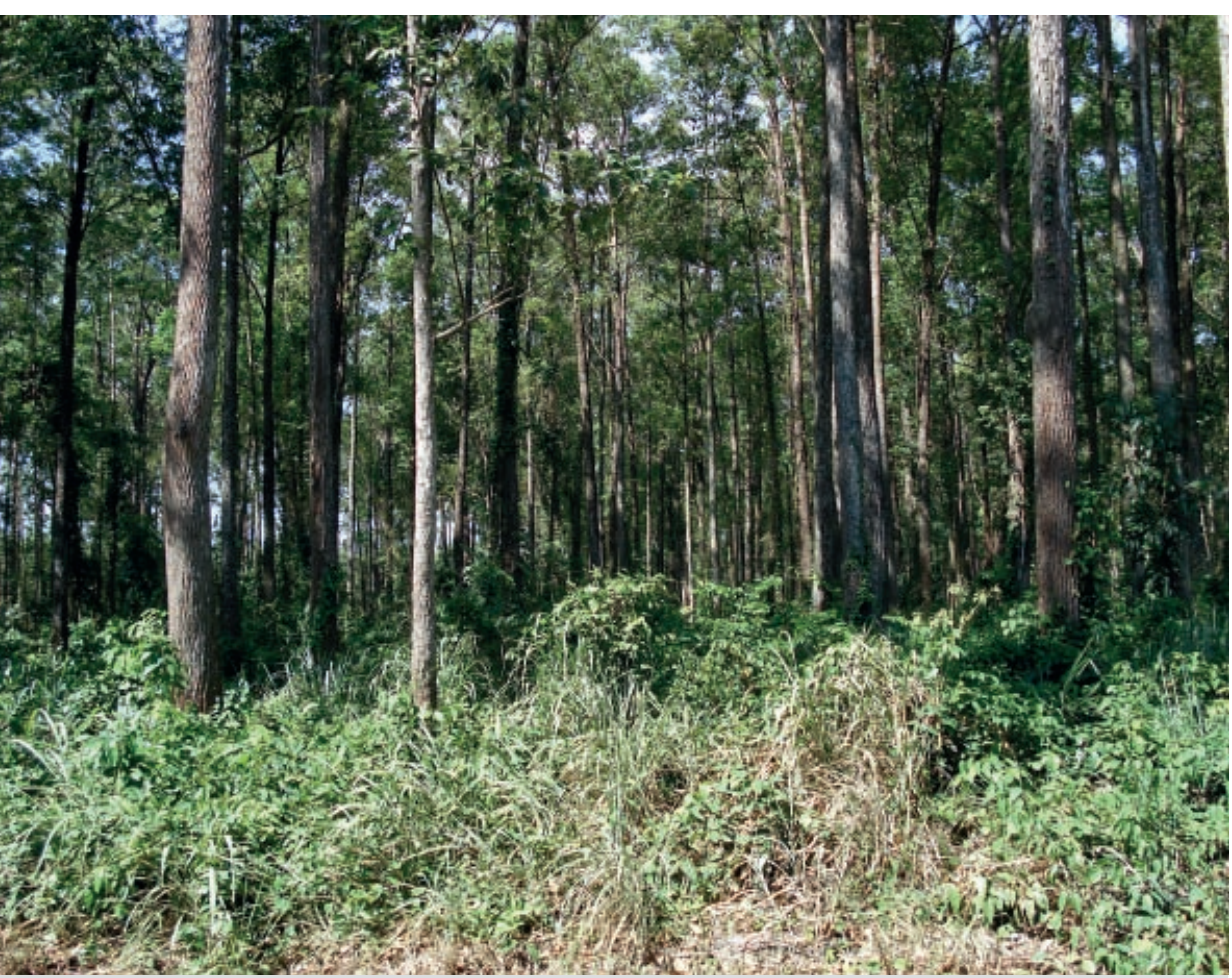

Sous-bois important dans une parcelle d'eucalyptus âgée de plus de 10 ans dans la région de Breu Branco. Photo M. Behling.
Des paramètres importants du modèle ont été estimés dans les neuf parcelles étudiées :

- La matière sèche de litière au sol a été évaluée dans chaque sous-parcelle à partir de quatre prélèvements aléatoires sur une surface de 0,28 $\mathrm{m}^{2}$, délimitée par un collecteur circulaire de 30 centimètres $(\mathrm{cm})$ de diamètre. - L'aire foliaire spécifique $(\sigma f)$ (aire foliaire par unité de matière sèche, $\mathrm{m}^{2} / \mathrm{kg}$ ) a été estimée sur 30 feuilles collectées aléatoirement aux quatre points cardinaux dans les tiers supérieur, moyen et inférieur du houppier. Ces feuilles fraîches ont été scannées (scanner Scanjet 3400C / Hewlett Packard) à une résolution de 200 points par pouce (dpi), l'analyse des images à partir du logiciel Matlab 6.0 permettant ensuite d'estimer précisément leur surface. Après séchage de ces feuilles à $65^{\circ} \mathrm{C}$, l'aire foliaire spécifique dans chaque tiers du houppier de chaque arbre a pu alors être estimée.

- La fraction de branches et écorce (pBB) par rapport à la biomasse ligneuse totale aérienne (sans les feuilles) et la densité du bois ont été déterminées pour chaque arbre échantillon. Des disques de $2 \mathrm{~cm}$ d'épaisseur ont été prélevés dans chacun des billons (tous les $3 \mathrm{~m}$ le long du tronc) afin d'évaluer la densité de bois $(\rho)$. Cette densité est calculée comme la moyenne des valeurs de densités mesurées pour chaque section, pondérée par la biomasse de bois de chaque section.

Les modèles non linéaires proposés par SANDS et LANDSBERG (2002) ont été ajustés pour évaluer les variations de $\sigma \mathrm{f}, \rho$ et $\mathrm{pBB}$, avec l'âge des peuplements (tableau II), en utilisant le logiciel Statistica 7.0.

Des mesures complémentaires pour évaluer la fertilité des parcelles ont été effectuées : des échantillons de sol ont été collectés sur les couches $0-20 \mathrm{~cm}$ et $20-40 \mathrm{~cm}$, séchés à l'air puis tamisés (maille de $2 \mathrm{~mm}$ ) pour analyses chimiques. Le stade nutritionnel des arbres a été évalué en collectant les deux premières feuilles entièrement développées ( $3^{\mathrm{e}}$ ou $4^{\mathrm{e}}$ paires de feuilles, soit 40 à 80 feuilles au total par sous-parcelle) sur des branches situées dans le tiers moyen ou supérieur du houppier, aux quatre points cardinaux. Les teneurs en azote $(N)$, phosphore $(P)$, potassium (K), calcium (Ca) et magnésium $(\mathrm{Mg})$ ont été déterminées en laboratoire pour un échantillon composite de feuilles par sous-parcelle.

Les précipitations moyennes recueillies sur une période de 10 ans ont été utilisées pour la phase de calibration du modèle. Les températures maximales et minimales ainsi 
que les radiations solaires globales ont été obtenues à partir des coordonnées géographiques, utilisant une banque de données climatiques disponible pour l'Amérique du Sud. Des données mises à disposition par l'entreprise et par la station météorologique la plus proche du site étudié ont également été utilisées.

\section{Calibration du modèle 3-PG}

La calibration a été effectuée à partir de la comparaison des données observées sur les parcelles étudiées aux données estimées par le modèle (LANDSBERG et al., 2003 ; ALMEIDA et al., 2004). Cette comparaison teste la capacité du modèle à simuler les variations de la croissance à court terme et à l'estimer en fin de rotation. Au cours de la calibration, les valeurs de certains paramètres ou variables non mesurés ont été modifiées pour réduire l'écart entre valeurs estimées et observées et ainsi obtenir un meilleur ajustement du modèle. Des simulations pour la matière sèche ligneuse, le diamètre et la hauteur moyenne, la surface terrière, le volume de bois et l'accroissement moyen annuel, ont été réalisées et comparées avec les mesures in situ.

L'ajustement entre données observées et simulées dans les peuplements étudiés a été réalisé pour les paramètres pour lesquels le modèle présente une forte sensibilité (LANDSBERG et al. 2003 ; ESPREY et al., 2004) : efficience maximale du houppier à convertir le rayonnement solaire en photoassimilats, conductance maximale du houppier, allocations de biomasse entre feuilles et biomasse ligneuse pour un diamètre de $20 \mathrm{~cm}$ du tronc à $1,30 \mathrm{~m}$, taux maximal de chute de litière et âge à partir duquel les chutes de litière se stabilisent.

\section{Simulations de scénarios contrastés quant à la disponibilité en eau et à la fertilité du sol}

Suite à l'ajustement du modèle 3-PG pour la région de Breu Branco et en considérant les estimations fournies par ce modèle cohérentes avec les données mesurées de croissance, des simulations ont été réalisées à partir de scénarios contrastés de disponibilité de facteurs de production (eau et nutriments). Ces simulations ont permis d'évaluer l'impact de ces scénarios sur le potentiel productif et de tester la sensibilité du modèle à différentes conditions hydriques et nutritionnelles. La température varie très peu tout au long de l'année mais la distribution annuelle des pluies est mal répartie, avec cinq mois ayant une pluviosité inférieure à $50 \mathrm{~mm}$ (figure 2). Quatre scénarios ont été simulés :

- pas de limitation hydrique à la croissance ;

- les précipitations distribuées de façon homogène (200 mm/mois durant toute l'année) ;

- les précipitations réelles ;

- les précipitations nulles durant la période de déficit hydrique.

Pour minimiser les écarts entre production observée et prédite, le paramètre fertilité du sol a été ajusté sur le massif en le fixant à la valeur 0,3, pour une carence minérale forte, et à la valeur 1 , pour des conditions nutritionnelles non limitantes.

\section{Principaux résultats concernant l'adaptation des eucalyptus à la zone d'étude}

\author{
Principales caractéristiques des plantations \\ de l'entreprise Dow Corning
}

Les analyses chimiques montrent que les sols sont acides, pauvres en éléments nutritifs et présentent une saturation élevée en aluminium (BouILLET et al., 2010). Les teneurs faibles en matière organique (MO) peuvent s'expliquer en partie par les pratiques culturales de l'entreprise, qui effectue des travaux du sol superficiels jusqu'à l'âge de trois ans pour éliminer le sous-bois, favorisant ainsi la décomposition de la MO (tableau I). Les faibles teneurs en éléments minéraux du sol dans ces parcelles ne sont pas compensées par des apports suffisants de fertilisants. Les analyses foliaires montrent que ces faibles fertilisations, associées à une compétition élevée entre eucalyptus et plantes adventices, entraînent des carences nutritionnelles (tableau III).

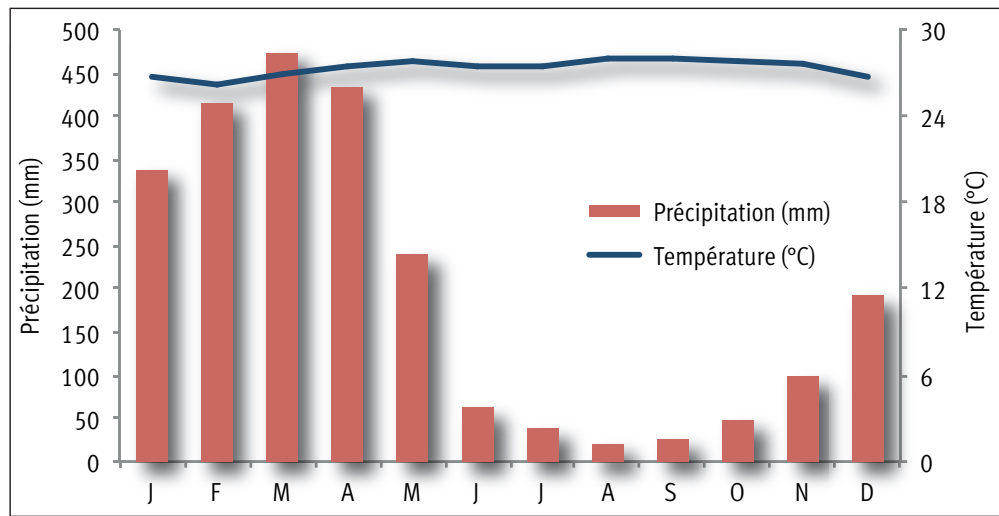

Figure 2.

Pluviométrie et températures mensuelles moyennes dans la région de Breu Branco.

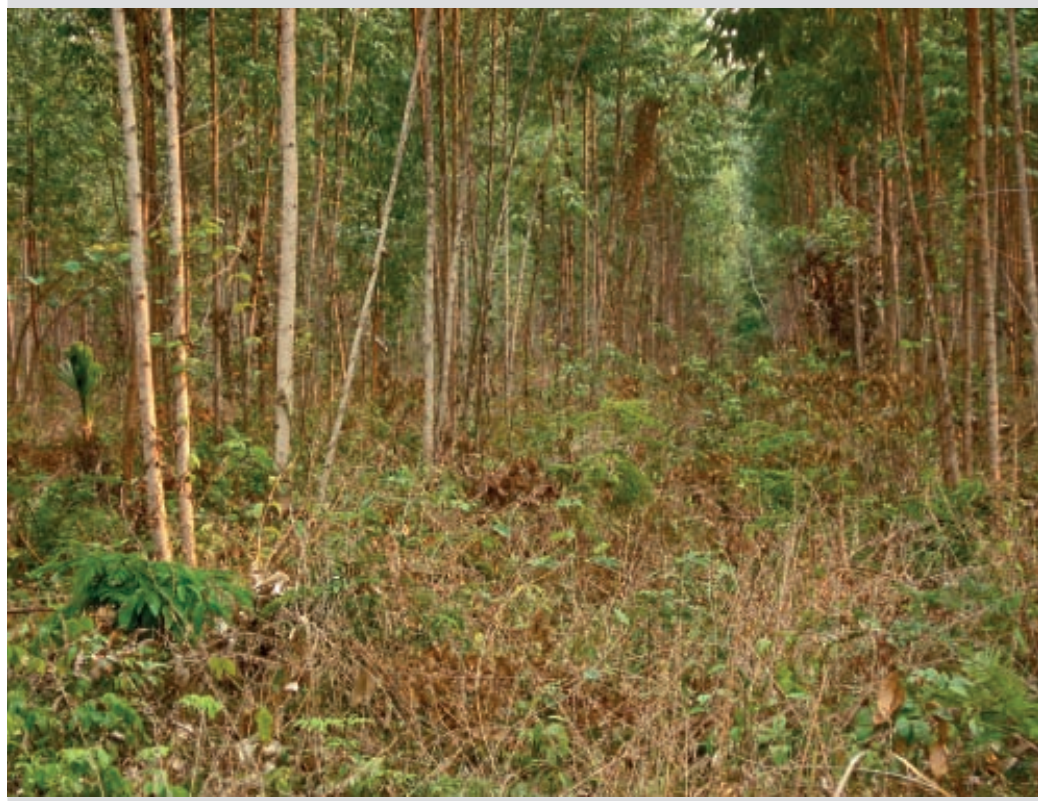

Sous-bois herbacé dense dans un taillis d'eucalyptus âgé d'environ un an qui limite fortement la croissance des eucalyptus. Photo M. Behling. 
Tableau III.

Teneurs en nutriments des feuilles d'eucalyptus.

\begin{tabular}{|c|c|c|c|c|c|c|c|c|c|c|c|c|c|c|}
\hline \multirow[t]{2}{*}{ Parcelle } & \multirow[t]{2}{*}{ Clone } & \multirow[t]{2}{*}{ Sous-parcelle } & $\mathrm{N}$ & $P$ & K & $\mathrm{Ca}$ & $\mathrm{Mg}$ & S & $B$ & $\mathrm{Cu}$ & $\mathrm{Fe}$ & $\mathrm{Mn}$ & $\mathrm{Zn}$ & $\mathrm{Na}$ \\
\hline & & & & & \multicolumn{2}{|c|}{$\mathrm{g} / \mathrm{kg}$} & & & & & \multicolumn{2}{|c|}{ mg/kgg } & & \\
\hline \multirow[t]{4}{*}{ T11 } & \multirow[t]{4}{*}{ PE-168 } & 1 & 18,5 & 1,09 & 7,0 & 3,1 & 0,7 & 0,5 & 17 & 7,0 & 62,0 & 240 & 11 & 730 \\
\hline & & 2 & 19,6 & 1,20 & 7,1 & 3,8 & 1,0 & 0,6 & 20 & 7,0 & 74,0 & 321 & 12 & 640 \\
\hline & & 3 & 19,0 & 1,14 & 7,3 & 3,1 & 1,8 & 0,6 & 19 & 7,0 & 50,0 & 277 & 12 & 650 \\
\hline & & 4 & 17,9 & 1,02 & 7,7 & 3,3 & 1,6 & 0,7 & 17 & 6,0 & 51,0 & 263 & 11 & 610 \\
\hline \multirow[t]{4}{*}{ T17 } & \multirow[t]{4}{*}{ PE-168 } & 1 & 17,9 & 1,20 & 4,9 & 5,2 & 2,2 & 0,5 & 18 & 7,0 & 41,0 & 179 & 16 & 600 \\
\hline & & 2 & 17,2 & 1,07 & 4,6 & 5,2 & 2,2 & 0,5 & 19 & 7,0 & 41,0 & 240 & 17 & 500 \\
\hline & & 3 & 18,8 & 0,98 & 5,9 & 4,8 & 2,8 & 0,6 & 22 & 7,0 & 42,0 & 154 & 13 & 50 \\
\hline & & 4 & 18,5 & 0,97 & 5,5 & 5,3 & 1,9 & 0,7 & 18 & 6,0 & 53,0 & 150 & 13 & 190 \\
\hline & & & & & & & & & & & & & & \\
\hline \multirow[t]{4}{*}{ T37 } & \multirow[t]{4}{*}{ UR-057 } & 1 & 13,9 & 0,79 & 4,5 & 3,7 & 2,1 & 0,6 & 24 & 5,0 & 39,0 & 249 & 12 & 890 \\
\hline & & 2 & 16,6 & 1,02 & 3,6 & 4,3 & 2,5 & 0,6 & 27 & 5,0 & 42,0 & 317 & 13 & 710 \\
\hline & & 3 & 15,3 & 0,94 & 4,0 & 4,1 & 2,2 & 0,5 & 23 & 6,0 & 38,0 & 301 & 13 & 640 \\
\hline & & 4 & 13,6 & 0,70 & 3,3 & 5,3 & 2,4 & 0,5 & 30 & 5,0 & 45,0 & 335 & 14 & 810 \\
\hline
\end{tabular}

La compétition pour l'eau et les nutriments entre les eucalyptus et les espèces pionnières de forêt naturelle est fréquemment observée dans la région et est probablement un facteur limitant majeur pour la croissance des arbres. A contrario, la diminution de cette compétition devrait améliorer sensiblement le statut nutritionnel des arbres, permettant ainsi un gain de productivité important.

\section{Calibration et étude des facteurs influençant la production}

Le tableau IV présente les valeurs des paramètres du modèle 3-PG adaptées aux conditions de croissance de la zone d'étude. Les résultats finaux, après calibration, comparant les caractéristiques dendrométriques estimées par le modèle avec les valeurs observées pour le clone UR-057, sont présentés dans la figure 3 .

L'ajustement du modèle aux conditions locales a permis d'améliorer considérablement les estimations de croissance de peuplements d'eucalyptus pour la région de Breu Branco. Les simulations montrent qu'une paramétrisation faite dans des conditions différentes de celles de la région d'étude ne permet pas d'estimer correctement la production de ces plantations, les différences pouvant être considérables (figure 4).

Les estimations de croissance dans la région de Breu Branco obtenues par le modèle 3-PG montrent que la production sans apport d'engrais ni contrôle des adventices serait seulement d'environ $70 \mathrm{~m}^{3} /$ ha en fin de rotation (figure 5, basse fertilité). Elles montrent également que des fertilisations soutenues - soit un apport par hectare en début de rotation d'environ 50 kilogrammes $(\mathrm{kg})$ de phosphore assimilable (hémipentoxyde de phosphore, $\mathrm{P}_{2} \mathrm{O}_{5}$ ), $200 \mathrm{~kg}$ de chlorure de potassium $(\mathrm{KCl})$ et $100 \mathrm{~kg}$ d'azote $(\mathrm{N})$ - pourraient permettre une augmentation de la productivité de l'ordre de 60 mètres cubes par hectare $\left(\mathrm{m}^{3} / \mathrm{ha}\right)$ par rapport aux pratiques culturales actuelles.

En outre, malgré une forte pluviosité annuelle, la disponibilité hydrique est le principal facteur limitant la croissance des eucalyptus dans la région en raison d'une saison sèche marquée dans certaines zones. Dans le cas d'un scénario comparant la productivité en fonction des précipitations observées et des précipitations nulles lors de la saison sèche de six mois, les estimations montrent, avec une fertilisation adéquate, une augmentation de seulement $34 \mathrm{~m}^{3} /$ ha au cours de la rotation (figure 5). En considérant des conditions non limitantes en éléments minéraux et en eau (avec des précipitations constantes tout au long de l'année de $200 \mathrm{~mm}$ par mois), cette augmentation serait nettement plus forte avec une production supérieure de $105 \mathrm{~m}^{3} /$ ha par rapport à celle obtenue avec les précipitations actuelles, et de $140 \mathrm{~m}^{3} /$ ha vis-à-vis du scénario où le déficit hydrique est aggravé (figure 5). La distribution des pluies plus régulière durant l'année induirait un net effet positif plus marqué sur les croissances estimées par le modèle que des précipitations totales plus importantes mais moins bien réparties. 
Tableau IV.

Paramétrisation du modèle 3-PG à partir des caractéristiques des plantations d'eucalyptus pour la région de Breu Branco.

Paramètres

Allocation feuille/bois peuplement jeune

Allocation feuille/bois peuplement adulte

Constante de la relation masse du tronc $x$ diamètre

Puissance de la relation masse du tronc $x$ diamètre

Fraction maximale de la production primaire nette pour les racines

Fraction minimale de la production primaire nette pour les racines

Taux de chute de litière à l'âge initial

Taux de chute de litière à l'âge adulte

Taux moyen de turnover racinaire

Température minimale pour la croissance

Température optimale pour la croissance

Température maximale pour la croissance

Niveau de fertilité

Réponse stomatique au déficit de pression de vapeur

Paramètre lié à la disponibilité hydrique du sol

Âge maximal du peuplement pour modéliser l'âge relatif

Conductance maximale du houppier

Indice surface foliaire LAI pour lequel la conductance du houppier est maximale

Conductance de la couche limite du houppier

Masse de bois maximale pour 1000 arbres par hectare

Aire foliaire spécifique pour peuplement d'âge adulte

Aire foliaire spécifique pour peuplement juvénile

Interception maximale de pluie par le houppier

LAl pour lequel l'interception d'eau par le houppier est maximale

Coefficient d'extinction de la radiation photosyntétiquement active

Âge de fermeture du couvert

Efficience quantique maximale du houppier

Relation entre production primaire nette et production primaire brute

Fraction de branches et écorce pour peuplement d'âge initial

Fraction de branches et écorce pour peuplement d'âge adulte

Densité basique minimale - peuplement d’âge initial

Densité basique maximale - peuplement d'âge adulte

\begin{tabular}{|c|c|c|c|}
\hline \multirow[t]{2}{*}{ Unité } & \multicolumn{3}{|c|}{ Coefficients } \\
\hline & UR-57 & PE-168 & $57+168$ \\
\hline - & 0,78 & 0,91 & 0,86 \\
\hline- & 0,11 & 0,13 & 0,11 \\
\hline- & 0,0613 & 0,0992 & 0,0789 \\
\hline- & 2,754 & 2,754 & 2,657 \\
\hline- & 0,8 & 0,8 & 0,8 \\
\hline- & 0,275 & 0,275 & 0,275 \\
\hline $1 /$ mois & 0,001 & 0,001 & 0,001 \\
\hline $1 /$ mois & 0,007 & 0,007 & 0,007 \\
\hline $1 /$ mois & 0,015 & 0,015 & 0,015 \\
\hline${ }^{\circ} \mathrm{C}$ & 8 & 8 & 8 \\
\hline${ }^{\circ} \mathrm{C}$ & 25 & 25 & 25 \\
\hline${ }^{\circ} \mathrm{C}$ & 40 & 40 & 40 \\
\hline- & $0,9-0,2$ & $0,9-0,2$ & $0,9-0,2$ \\
\hline $\mathrm{m} / \mathrm{bar}$ & 0,0324 & 0,0324 & 0,0324 \\
\hline- & Variable & Variable & Variable \\
\hline Année & 9 & 9 & 9 \\
\hline $\mathrm{m} / \mathrm{s}$ & 0,02 & 0,02 & 0,02 \\
\hline $\mathrm{m}^{2} / \mathrm{m}^{2}$ & 3,33 & 3,33 & 3,33 \\
\hline $\mathrm{m} / \mathrm{s}$ & 0,2 & 0,2 & 0,2 \\
\hline $\mathrm{kg} / \mathrm{arv}$ & 300 & 300 & 300 \\
\hline $\mathrm{m}^{2} / \mathrm{kg}$ & 6,96 & 8,13 & 7,55 \\
\hline $\mathrm{m}^{2} / \mathrm{kg}$ & 12,08 & 12,83 & 12,84 \\
\hline- & 0,15 & 0,15 & 0,15 \\
\hline $\mathrm{m}^{2} / \mathrm{m}^{2}$ & 3,33 & 3,33 & 3,33 \\
\hline- & 0,47 & 0,47 & 0,47 \\
\hline Année & 1,5 & 1,5 & 1,5 \\
\hline- & 0,08 & 0,08 & 0,08 \\
\hline- & 0,47 & 0,47 & 0,47 \\
\hline- & 0,16 & 0,16 & 0,17 \\
\hline- & 0,58 & 0,55 & 0,58 \\
\hline $\mathrm{t} / \mathrm{m}^{3}$ & 0,390 & 0,430 & 0,390 \\
\hline $\mathrm{t} / \mathrm{m}^{3}$ & 0,520 & 0,540 & 0,530 \\
\hline
\end{tabular}

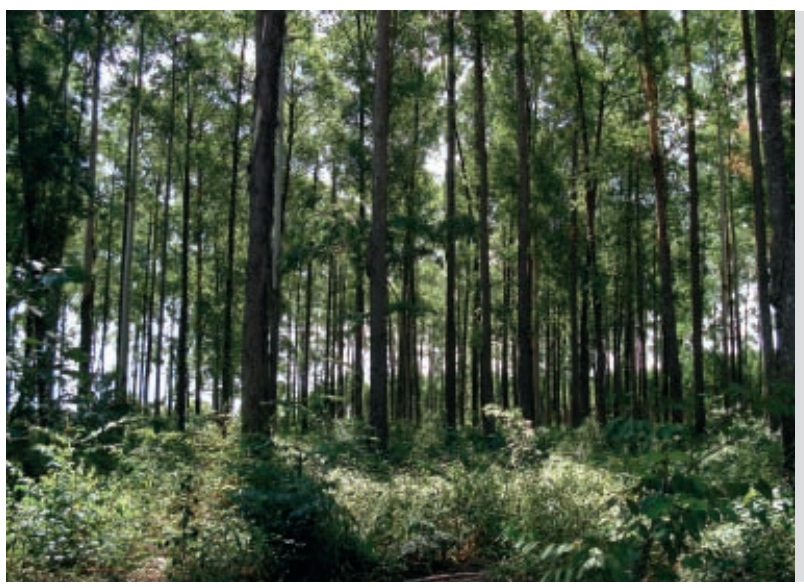

Parcelle de conservation de matériel génétique d'eucalyptus adapté aux conditions environnementales de la région de Breu Branco.

Photo M. Behling. 

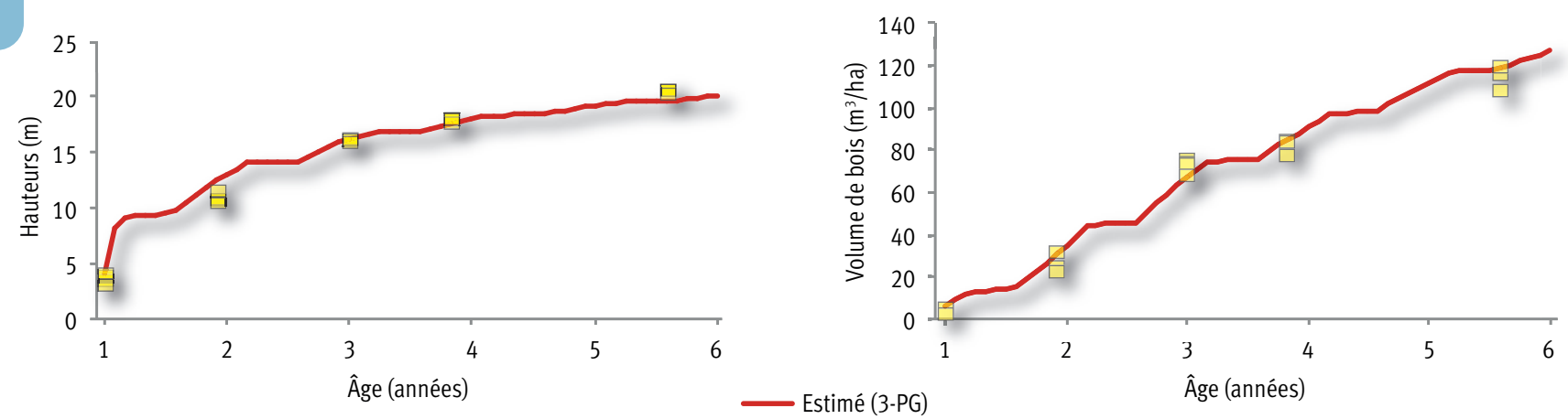

$\square$ Observé

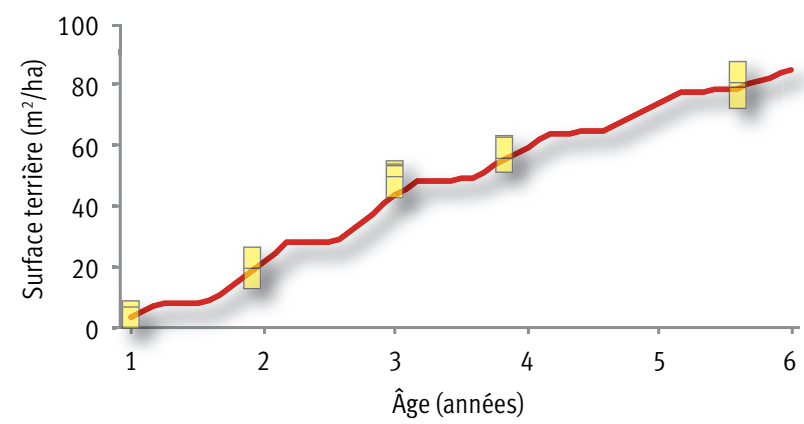

Figure 3.

Estimations de hauteur moyenne, surface terrière, volume de bois et accroissement moyen annuel (AMA), obtenues

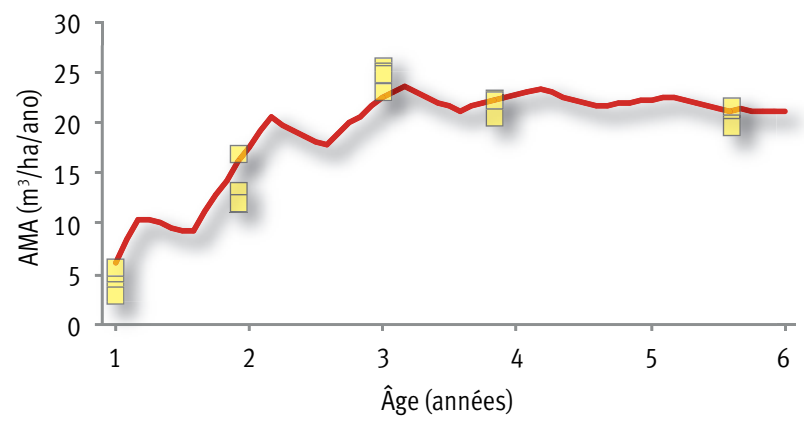

par le modèle 3-PG. Ces prédictions sont comparées aux données observées pour le clone UR-57, de 1,0 à 5,6 ans, pour la région de Breu Branco.
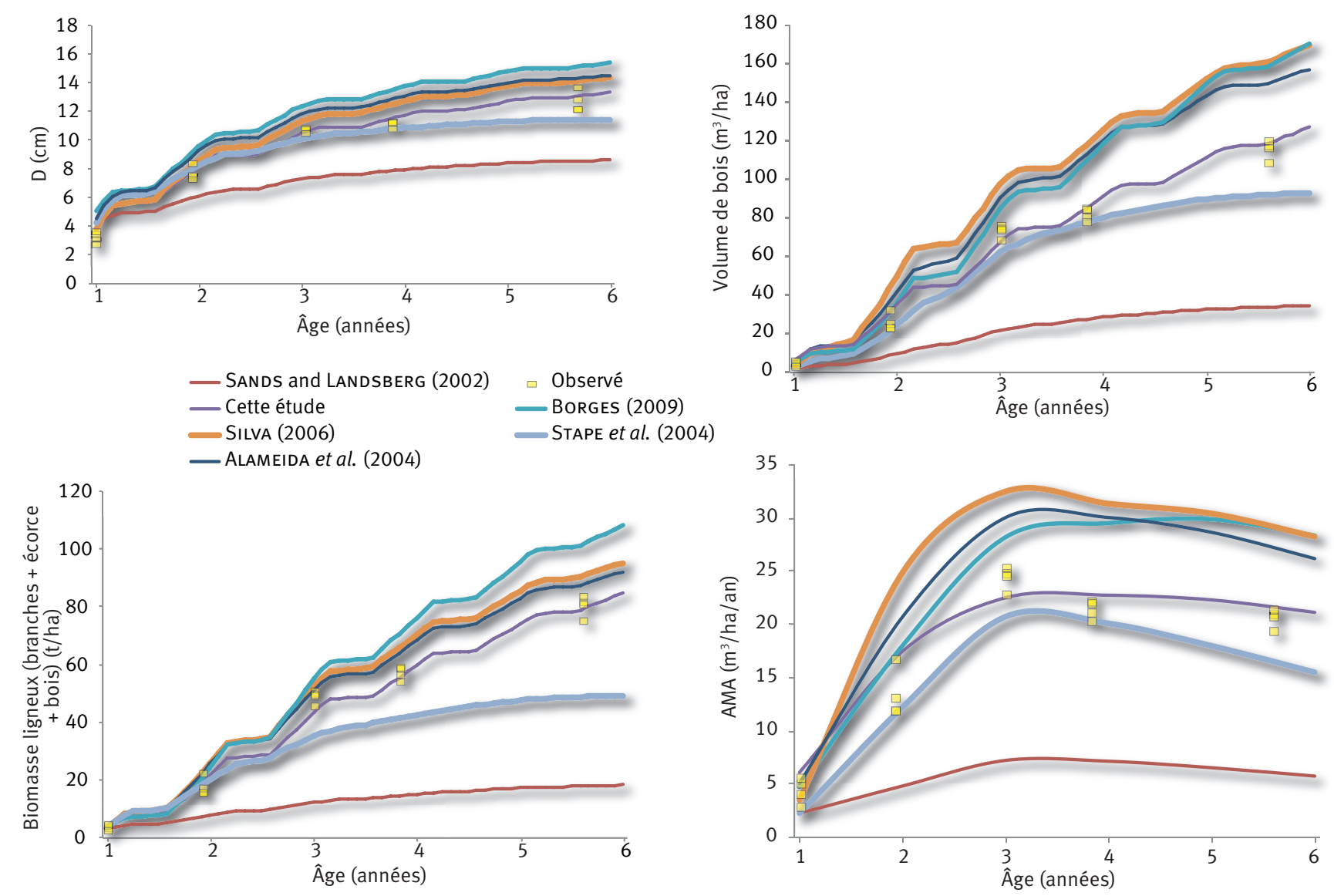

Figure 4.

Prédictions par le modèle 3-PG du diamètre moyen des arbres (D), de la biomasse ligneuse, du volume total du tronc et de l'accroissement moyen annuel (AMA), pour des valeurs de paramètres en plantations d'eucalyptus

indiquées dans la littérature. Les simulations effectuées de 1 à 6 ans pour le clone UR-57 avec la calibration effectuée localement sont également indiquées, ainsi que les valeurs mesurées pour les plantations étudiées. 

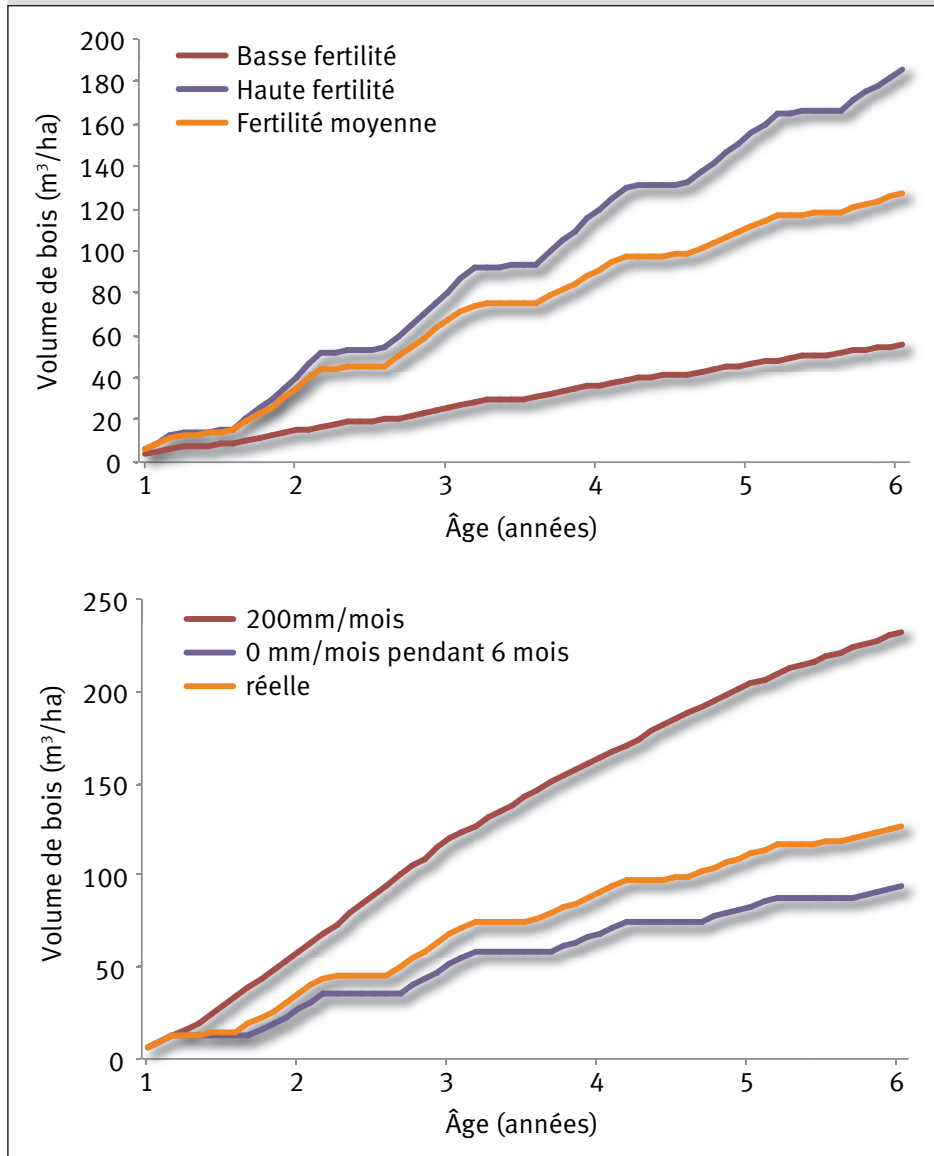

Figure 5.

Simulations de différents scénarios de fertilité du sol et de pluviométrie hydrique sur le volume de bois avec la paramétrisation de 3-PG faite pour le clone UR-57, pour la région de Breu Branco.

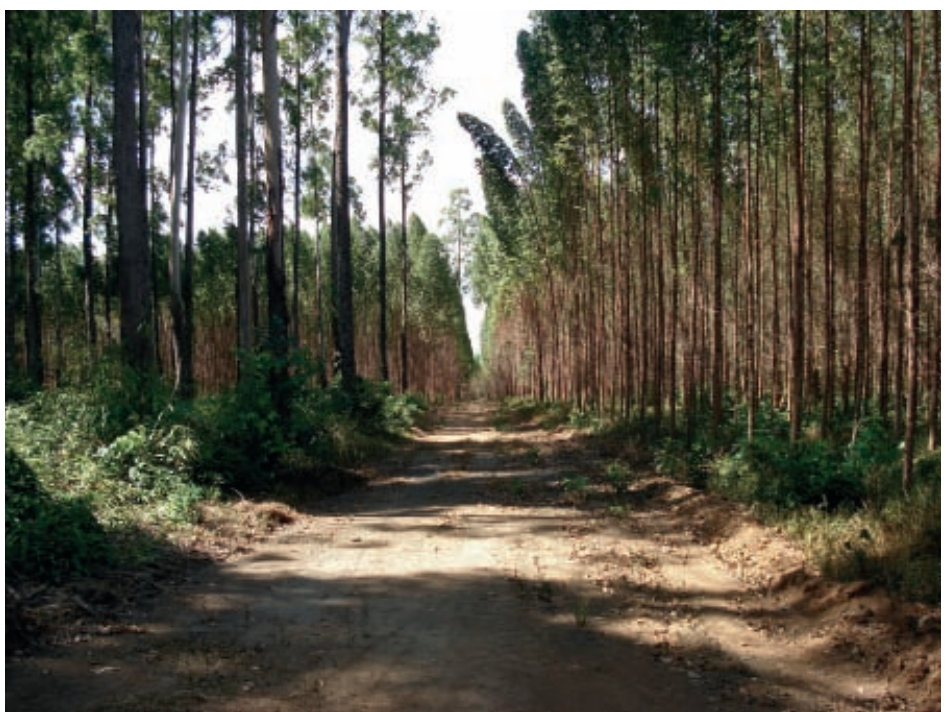

Parcelles commerciales de différents âges. Le sous-bois abondant limite fortement la croissance des arbres et la production de bois. Photo M. Behling.

\section{Discussion}

L'étude a montré que du matériel végétal adapté à la région existe en termes de croissance et qu'il présente des caractéristiques de densité du bois intéressantes pour la production de charbon (BoulLLeT et al., 2010). La pluviométrie varie assez peu dans un rayon de 300 kilomètres autour du pôle de Carajás, mais les entreprises devront privilégier pour les futures plantations les zones où la saison sèche est la moins marquée. En effet, les résultats du modèle montrent que la distribution des pluies influence considérablement la production des plantations commerciales dans cette zone.

Les simulations réalisées avec le modèle 3-PG ont montré que la sylviculture a un impact marqué sur la production. Les opérations sylvicoles à mener sont en priorité : le soussolage avant plantation en sol argileux ou en présence d'une couche de sol empêchant le développement racinaire en profondeur ; des passages réguliers d'herbicides afin de contrôler efficacement le sous-bois herbacé et ligneux très envahissant dans cette région et très compétitif vis-à-vis des eucalyptus pour l'absorption de l'eau et des éléments nutritifs ; des fertilisations suffisantes en N, P, K, Ca et Mg car les réserves minérales sont très faibles dans ces sols fortement altérés. Des évaluations des coûts de production de charbon de bois à partir de biomasse issue de plantations d'eucalyptus ont été réalisées (Morello, PIKETTY, 2010).

Les plantations d'eucalyptus doivent aussi répondre à un certain nombre de préoccupations, particulièrement en matière d'impacts environnementaux et socio-économiques. Ces plantations peuvent en effet avoir des impacts très négatifs sur l'environnement si elles sont mises en place après déforestation de forêts naturelles (pertes de biodiversité, émission nette de carbone...). En revanche, l'impact environnemental de plantations très productives installées sur de faibles surfaces de pâturages dégradés n’ayant pas vocation à être régénérés en forêts naturelles, comme dans la région étudiée, peut être très limité.

Plus spécifiquement, les recherches sur les cycles de l'eau, du carbone et des éléments minéraux conduites au Congo et au Brésil (NouvelLon et al., 2009 ; LACLAu et al., 2010 ; BouILLET et al., 2010) permettent de mieux comprendre le fonctionnement de ces plantations et de les gérer en limitant leurs impacts environnementaux.

L'influence des eucalyptus sur les ressources en eau dépend des conditions écologiques locales, de la productivité des plantations, mais aussi de l'efficience d'utilisation de l'eau du matériel végétal utilisé. La transpiration maximale des plantations d'eucalyptus est d'environ 1500 mm/an après la phase d'installation des peuplements. Dans la région du pôle sidérurgique de Carajás, les précipitations annuelles sont supérieures à $1500 \mathrm{~mm}$ sauf dans certaines zones du Maranhão (comprises entre 1200 et $1500 \mathrm{~mm}$ ), ce qui est compatible avec l'alimentation des nappes phréatiques et/ou des rivières. La minimisation des impacts négatifs de ces plantations sur les ressources en eau est possible : soit en développant du matériel végétal ayant une efficience d'utilisation de l'eau élevée, soit grâce à des itinéraires techniques limitant la concurrence hydrique et l'évaporation par la végétation adventice, ou encore par un 


\section{Références bibliographiques}

ALMEIDA A., LANDSBERG L., SANDS P. J., 2004. Parameterisation of 3-PG model for fast-growing Eucalyptus grandis plantations. Forest Ecology and Management, 193: 179-195.

BATTAGLIA M., SANDS P., 1997. Modelling site productivity of Eucalyptus globulus in response to climatic and site factors. Australian Journal of Plant Physiology, 24: 831-850.

BOUILLET J.-P., LACLAU J.-P., BEHLING M., 2010. Projet Énergie et reboisement en Amazonie. Évaluation de l'intérêt écologique de différentes modalités de reboisement à vocation énergétique. Rapport final de la composante 2 écologique du projet Era. Montpellier, France, Cirad.

CALAIS D., 2009. Florestas energéticas no Brasil. Demanda e disponibilidade. Associação Mineira de Silvicultura, Belo Horizonte, 23 p. www.silviminas.com.br/Publicacao /Arquivos/publicacao_472.pdf (page consultée le 19 avril 2010).

COOPS N. C., WARING R. H., 2001. Assessing forest growth across southwestern Oregon under a range of current and future global change scenarios using a process model, 3-PG. Global Change Biology, 7: 15-29.

DYE P. J., 2004. Verification of 3-PG growth and water-use predictions in twelve Eucalyptus plantations stands in Zululand, South Africa. Forest Ecology and Management, 193: 197-218.

ESPREY L. J., SANDS P. J., SMITH C. W., 2004. Understanding 3-PG using a sensitivity analysis. Forest Ecology and Management, 193: 235-250.

FORRESTER D. I., BAUHUS J., COWIE A. L., VANCLAY J. K., 2006. Mixed-species plantations of Eucalyptus with nitrogen-fixing trees: a review. Forest Ecology and Management, 233: 211-230.

GUNES A., INAL N., CICEK N., ERASLAN F., 2007. Role of phosphatases, iron reducing, and solubilizing activity on the nutrient acquisition in mixed cropped peanuts and barley. Journal of Plant Nutrition, 30 (10-12): 1555-1568.

JUDD T. S., ATTIWILL P. M., ADAMS A. M., 1996. Nutrient concentration in Eucalyptus: A synthesis in relation to diferences between taxa, sites and components. In : Attiwill P. M., Adams M. A. (éd.). Nutrition of Eucalyptus. Melbourne, Australie, Csiro, $440 \mathrm{p}$.

LACLAU J.-P., RANGER J., DE MORAES GONÇALVES J. L., MAQUÈRE V., KRUSCHE A. V., THONGO M'BOU A., NOUVELLON Y., SAINT-ANDRÉ L., BOUILLET J.-P., DE CASSIA PICCOLO M., DELEPORTE P., 2010. Biogeochemical cycles of nutrients in tropical Eucalyptus plantations. Main features shown by intensive monitoring in Congo and Brazil. Forest Ecology and Management, 259: 1771-1785.

LANDSBERG J. J., WARING R. H., COOPS N. C., 2003. Performance of the forest productivity model 3-PG applied to a wide range of forest types. Forest Ecology and Management, 172: 199-214.
MALAVOLTA E., VITTI G. C., OLIVEIRA S. A., 1997. Avaliação do estado nutricional das plantas: princípios e aplicações. 2 ed. Piracicaba, Brésil, Associação Brasileira para a Pesquisa da Potassa e do Fosfato, 319 p.

MORELLO T. F., PIKETTY M. G., 2010. Transição das siderurgias de Carajás para um padrão de baixa contestabilidade socioambiental: foco nos incentivos econômicos. Energia e reflorestamento na Amazônia (ERA), Relatorio final componente 2. www.nesa.org.br/pdf/ Consultorias/Morello\%20_ \%20Piketty\%20_2010_\%20final.pdf (page consultée le $1^{\text {er }}$ avril 2010).

NOUVELLON Y., STAPE J. L., BONNEFOND J.-M., BOUILLET J.P., SAINT-ANDRÉ L., HAMEL O., EPRON D., LE MAIRE G., ROUPSARD O., DA ROCHA H., GONÇALVES J. L. M., MARSDEN C., JOURDAN C., LACLAU J.-P., 2009. Carbon sequestration and water-use by eucalypt plantations in Congo and Brazil. Communication à la Conférence internationale " La science au service de la gestion des forêts tropicales humides ", 22 28 novembre 2009, Cayenne, Guyane française.

PIKETTY M. G., MORELLO T. F., BOUILLET J.-P., LACLAU J.-P., BEHLING M., PIRES A. C., ROUSSET P., RODRIGUES T., DUFOUR T., DURIEUX L., SIST P., VIEIRA P., 2011. Charbon de bois et sidérurgie en Amazonie brésilienne : quelles pistes d'améliorations environnementales ? L'exemple du pôle de Carajás. Paris, France, Agence française de développement, Document de travail $n^{\circ} 112$.

SANDS P. J., LANDSBERG J. J., 2002. Parametrisation of 3-PG for plantation grown Eucalyptus globulus. Forest Ecology and Management, 163: 273-292.

SANDS P. J., 2004. 3-PGPJS vsn 2.4 - a user-friendly interface to 3-PG, the Landsberg and Waring model of forest productivity. Hobart, Australie, CRC for Sustainable Production Forestry, CSIRO Forestry and Forest Products, Technical Report, $n^{\circ}$ 140. www.landsberg.com.au, www.ffp.csiro.au SINDIFER, 2010. Produção Total de Ferro-Gusa no Brasil, 2007. 1 p. www.sindifer.com.br/Anuario_2007.html (page consultée le 5 octobre 2010).

STAPE J. L., RYAN M. G., BINKLEY D., 2004. Testing the utility of the 3-PG model for growth of Eucalyptus grandis $x$ E. urophylla with natural and manipulated supplies of water and nutrients. Forest Ecology and Management, 193: 219-234. 